\title{
OPEN The influence of sex and body mass index on the association between soluble neprilysin and risk of heart failure hospitalizations
}

\author{
Julio Núñez $\mathbb{1}^{1,2 凹}$, Eduardo Núñez ${ }^{1}$, Elena Revuelta-López ${ }^{2,3}$, Gema Miñana ${ }^{1,2}$, \\ Jaume Barallat ${ }^{3}$, Vicent Bodii, ${ }^{1}$, Juan Sanchis ${ }^{1,2}$, Alberto Aimo ${ }^{4,5}$, Michele Emdin ${ }^{5,6}$, \\ Josep Lupón ${ }^{2,7,8}$, Oliver Husser ${ }^{9}$ \& Antoni Bayes-Genis ${ }^{2,7,8}$
}

\begin{abstract}
A higher neprilysin activity has been suggested in women. In this retrospective analysis, we evaluated the association of sex and body mass index (BMI) with soluble neprilysin (sNEP) and recurrent admissions among 1021 consecutive HF outpatients. The primary and secondary endpoints were the number of HF hospitalizations and all-cause mortality, respectively. The association between SNEP with either endpoint was evaluated across sex and BMI categories $\left(\geq 25 \mathrm{~kg} / \mathrm{m}^{2} \mathrm{vs} .<25 \mathrm{~kg} / \mathrm{m}^{2}\right)$. Bivariate count regression (Poisson) was used, and risk estimates were expressed as incidence rates ratio (IRR). During a median follow-up of 6.65 years (percentile 25\%-percentile 75\%:2.83-10.25), 702 (68.76\%) patients died, and $406(40 \%)$ had at least $1 \mathrm{HF}$ hospitalization. Median values of SNEP and BMI were $0.64 \mathrm{ng} / \mathrm{mL}(0.39-1.22)$, and $26.9 \mathrm{~kg} / \mathrm{m}^{2}(24.3-30.4)$, respectively. Left ventricle ejection fraction was $<40 \%$ in $78.9 \%$ of patients, and $28 \%$ were women. In multivariable analysis, sNEP (main effect) was positively associated with HF hospitalizations $(p=0.001)$ but not with mortality $(p=0.241)$. The predictive value of $s$ NEP for HF hospitalizations varied non-linearly across sex and BMI categories ( $p$-value for interaction $=0.003$ ), with significant and positive effect only on women with BMI $\geq 25 \mathrm{~kg} /$ $\mathrm{m}^{2}(p=0.039$ ). For instance, compared to men, women with sNEP of $1.22 \mathrm{ng} / \mathrm{mL}$ (percentile $75 \%$ ) showed a significantly increased risk (IRRs: $1.26 ; 95 \% \mathrm{Cl}$ : 1.05-1.53). The interaction analysis for mortality did not support a differential prognostic effect for $\operatorname{sNEP}(p=0.072)$. In conclusion, higher sNEP levels in overweight women better predicted an increased risk of HF hospitalization.
\end{abstract}

The enzyme neprilysin breaks down various vasoactive peptides, including natriuretic peptides, and plays a key role in the pathophysiology of heart failure $(\mathrm{HF})^{1}$. Over the last few years, neprilysin inhibition has become a therapeutic target in patients with $\mathrm{HF}^{2,3}$. Subgroup analyses from randomized clinical trials indicate that neprilysin inhibition by sacubitril/valsartan may decrease the risk of recurrent hospitalization in women with HF with preserved ejection fraction ${ }^{4,5}$. Although some authors suggest that neprilysin activity is higher in women with $\mathrm{HF}^{6,7}$, the evidence explaining the sex-differential findings remains mostly speculative.

The circulating soluble form of the extracellular domain of neprilysin (sNEP) has emerged as a promising biomarker in patients with acute and chronic HF for predicting cardiovascular death and hospitalizations ${ }^{8-11}$, despite conflicting results between groups and different immunoassay utilized ${ }^{12,13}$. It is currently unclear whether the association between sNEP and hospitalization burden is modified by sex and body mass index (BMI). Therefore, we evaluated the influence of sex and BMI on the prognostic value of sNEP in patients with HF.

\footnotetext{
${ }^{1}$ Cardiology Department, Hospital Clínico Universitario, INCLIVA, Departamento de Medicina, Universitat de València, Avda. Blasco Ibáñez 17, 46010 Valencia, Spain. ${ }^{2}$ CIBER Cardiovascular, Madrid, Spain. ${ }^{3}$ Biochemistry

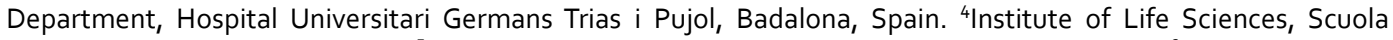
Superiore Sant'Anna, Pisa, Italy. ${ }^{5}$ Cardiology Division, University Hospital of Pisa, Pisa, Italy. ${ }^{6}$ Scuola Superiore Sant'Anna, Pisa, Italy. ${ }^{7}$ Heart Failure Unit, Cardiology Department, Hospital Universitari Germans Trias i Pujol, Badalona, Spain. ${ }^{8}$ Department of Medicine, Autonomous University of Barcelona, Barcelona, Spain. ${ }^{9} \mathrm{Klinik}$ für Innere Medizin I, Kardiologie, St. Johannes-Hospital Dortmund, Dortmund, Germany. ${ }^{\square}$ email: yulnunez@ gmail.com
} 


\section{Methods}

Study population. This is an observational retrospective analysis from a prospective registry carried on in a third-level university hospital ${ }^{11}$. We analyzed 1021 consecutive ambulatory patients evaluated from May 2006 to May 2013 at a multidisciplinary HF clinic. Data on patient demographics, medical history, physical examination, 12-lead electrocardiogram, laboratory tests, echocardiogram, and medications were included in pre-established electronic questionnaires. The principal referral criterion was HF diagnosis, according to the European Society of Cardiology guidelines ${ }^{3}$, with at least one hospitalization for acute heart failure (AHF) or reduced left ventricular ejection fraction (LVEF). No patient was on sacubitril/valsartan or other NEP inhibitors.

BMI was calculated in all patients using calibrated scales. The associations between sNEP and the outcomes were evaluated in the whole population and stratified patients according to sex and BMI (considering the standard cut-off of $25 \mathrm{~kg} / \mathrm{m}^{2}$ used to categorize normal weight from overweight) ${ }^{14}$.

All participants provided written informed consent, and the local ethics committee (Comité dètica de la Investigació Clínica del Hospital Universitari Germans Trias i Pujol) approved the study. The study protocol conformed to the ethical guidelines of the 1975 Declaration of Helsinki (revised in 1983), as reflected by an a priori approval by the institution's human research committee. Patients were not involved in the design and conduct of this research.

sNEP measurement. All blood samples were drawn between 09:00 a.m. and noon and stored as plasma at $-80{ }^{\circ} \mathrm{C}$, without previous freeze-thaw cycles. We measured sNEP by a modified sandwich immunoassay (human neprilysin/CD10 ELISA kit, Aviscera Bioscience, Santa Clara, California, code No. SK00724-01, lot No. 20111893). To improve the analytical sensitivity of the method and obtain a lower limit of sample quantification, several modifications were made: serum aliquots were diluted one-quarter before incubation in dilution buffer provided by the manufacturer (DB09); the kit was transferred to an automated robotic platform (Basic Radim Immunoassay Operator 2 [BRIO 2], Radim SpA, Pomezia, Italy) that performed all incubations at a constant temperature of $30^{\circ} \mathrm{C}$, with 1,000 revolutions/min mixing; and the initial sample incubation was extended to $150 \mathrm{~min}$ to achieve a higher slope in the calibration curve and better assay sensitivity.

The assay measures the 52 to a 750 -amino-acid fraction of neprilysin as an immunogen (extracellular soluble fraction). This assay has $0 \%$ cross-reactivity with the two metallopeptidases most similar to this sequence, endothelin converting enzymes 1 and 2 . The test also does not exhibit cross-reactivity with erythrocyte cellsurface antigen (KELL), another protein with strong homology with neprilysin. The modified protocol presented analytical linearity from 0.250 to $4 \mathrm{ng} / \mathrm{mL}$. Samples with concentrations $>4 \mathrm{ng} / \mathrm{mL}$ were further diluted to a final range of 0.250 to $64 \mathrm{ng} / \mathrm{mL}$. At a positive control value of $1.4 \mathrm{ng} / \mathrm{mL}$, the intra-assay and inter-assay coefficients of variation were $3.7 \%$ and $8.9 \%$, respectively. The intra-assay coefficient of variation at the median value $(0.642 \mathrm{ng} / \mathrm{mL})$ was $6.5 \%$.

Follow-up and outcomes. We followed up all patients at regular predefined intervals, with additional visits when medically necessary ${ }^{11}$. These scheduled visits included, at a minimum, quarterly visits with nurses, biannual visits with physicians, and elective visits with geriatricians, psychiatrists, and rehabilitation physicians. Patients who did not attend the regular visits were contacted by telephone ${ }^{11}$. We selected the total number of unplanned HF-related hospitalizations as the primary endpoint. We identified hospitalizations from patients' clinical records in the HF unit and hospital wards through the electronic Catalan medical record database. All-cause mortality was selected as a secondary endpoint. We identified fatal events from the HF unit's clinical records, hospital wards, emergency room, general practitioners and by contacting the patients' relatives. We verified the reliability of the data by double-checking the databases of the Catalan and Spanish health systems. For this study, the personnel in charge of clinical management and endpoint adjudication were unaware of the patient's sNEP level.

Statistical analysis. Continuous variables are expressed as mean \pm standard deviation (SD) or median [percentile 25\% (p25\%) to percentile 75\% (p75\%)] per variable distribution. Discrete variables are presented as percentages. Baseline characteristics based on sex and BMI were compared by ANOVA, Kruskal-Wallis, or chi-squared tests, as appropriate. Rates of events were presented as per 1 person-years (P-Y). To account for the positive correlation between HF hospitalization and mortality, we fitted the Famoye bivariate Poisson regression model, where the number of admissions (as counts) and mortality (as the terminal event) were modeled simultaneously and linked by shared frailty ${ }^{15}$. To account for differences in each recurrent event, the log of follow-up time was included as an offset in each submodel. Crude and adjusted rates (number of events per 1 person-year) are presented among the groups tested. We selected explanatory variables for the initial multivariable model based on subject-matter knowledge. Then, using a backward elimination (BE) procedure which includes a fractional polynomial (FP) transformation for continuous variables ${ }^{14}$, we arrived to a final model. In some instances, however, the automatic selection procedure was overridden by leaving important known predictors in HF's setting independent of their level of significance. For HF hospitalization, the best sNEP polynomial transformation was FP[-0.5]. The sNEP trajectories across sex/BMI categories are depicted in graphs after back-transformation to its original scale. The final covariates (and its FP transformation in case of continuous variables) included in the HF-rehospitalization model were age (FP:1), HF duration (FP:1), New York Heart Association (NYHA) class III versus I-II, ischaemic heart disease, systolic blood pressure (FP:1), heart rate (FP:2), serum sodium (FP:3), N-terminal pro-B-type natriuretic peptide (NT-proBNP) (FP:0), estimated glomerular filtration rate [Modification of Diet in Renal Disease (MDRD) formula] (FP:1), high-sensitivity troponin T (FP:0), LVEF $<40 \%$ versus $\geq 40 \%$, and treatment with angiotensin-converting enzyme inhibitors (ACEI) or angiotensin II receptor blockers (ARB), beta-blockers, mineralocorticoid receptor antagonists (MRA), or loop diuretics. For all-cause 


\begin{tabular}{|c|c|c|c|c|c|}
\hline Variables & $\begin{array}{l}\text { Women, } \text { BMI }<25 \mathrm{~kg} / \mathrm{m}^{2} \\
(\mathrm{n}=\mathbf{8 6})\end{array}$ & $\begin{array}{l}\text { Women, BMI } \geq 25 \mathrm{~kg} / \mathrm{m}^{2} \\
(\mathrm{n}=200)\end{array}$ & Men, $B M I<25 \mathrm{~kg} / \mathrm{m}^{2}(\mathrm{n}=234)$ & Men, $B M I \geq 25 \mathrm{~kg} / \mathrm{m}^{2}(\mathrm{n}=501)$ & $p$-value \\
\hline \multicolumn{6}{|c|}{ Demographics and medical history } \\
\hline Age, years & $69 \pm 14$ & $70 \pm 10$ & $67 \pm 14$ & $64 \pm 12$ & $<0.01$ \\
\hline BMI, $\mathrm{kg} / \mathrm{m}^{2 *}$ & $22.9(21.2,24.2)$ & $29.9(27.1,33.8)$ & $23.1(21.6,24.2)$ & $28.6(26.7,31.2)$ & $<0.01$ \\
\hline Hypertension, n (\%) & $52(60.5)$ & $153(76.5)$ & $125(53.4)$ & $307(61.3)$ & $<0.01$ \\
\hline DM, n (\%) & $24(27.9)$ & $90(45.0)$ & $59(25.2)$ & $187(37.3)$ & $<0.01$ \\
\hline Dyslipidemia, n (\%) & $31(33.0)$ & $88(44.0)$ & $96(41)$ & $266(53.1)$ & $<0.01$ \\
\hline Current smoker, n (\%) & $8(9.3)$ & $8(4.0)$ & $49(20.9)$ & $98(19.6)$ & $<0.01$ \\
\hline Former smoker, n (\%) & $9(10.5)$ & $15(7.5)$ & $126(53.8)$ & $276(55.1)$ & $<0.01$ \\
\hline COPD, n (\%) & $4(4.6)$ & $21(10.5)$ & $45(19.2)$ & $104(20.9)$ & $<0.01$ \\
\hline Ischemic etiology, n (\%) & $26(30.27)$ & 75 (37.5) & $134(57.3)$ & $286(57.1)$ & $<0.01$ \\
\hline HF duration, months ${ }^{*}$ & $24(2,72)$ & $24.3(6,70.3)$ & $17.2(2,60)$ & $28(4,72)$ & $<0.01$ \\
\hline ICD, n (\%) & $5(5.8)$ & $17(8.5)$ & $31(13.2)$ & $83(16.6)$ & $<0.01$ \\
\hline CRT, n (\%) & $4(4.5)$ & $14(7.0)$ & $16(6.8)$ & $51(10.2)$ & 0.178 \\
\hline NYHA class I or II, n (\%) & $57(66.3)$ & $133(66.5)$ & $177(75.6)$ & $406(81.0)$ & $<0.01$ \\
\hline LVEF $<40 \%, \mathrm{n}(\%)$ & $54(62.8)$ & $124(62.0)$ & $197(84.2)$ & $431(86.0)$ & $<0.01$ \\
\hline \multicolumn{6}{|l|}{ Vital signs } \\
\hline Heart rate, bpm & $75 \pm 17$ & $75 \pm 14$ & $71 \pm 14$ & $71 \pm 14$ & 0.07 \\
\hline SBP, mmHg & $126 \pm 23$ & $132 \pm 25$ & $125 \pm 24$ & $126 \pm 20$ & $<0.01$ \\
\hline \multicolumn{6}{|l|}{ Laboratory } \\
\hline Haemoglobin, g/dL & $12.0 \pm 1.7$ & $12.0 \pm 1.5$ & $13.1 \pm 2.0$ & $13.2 \pm 1.9$ & $<0.01$ \\
\hline $\begin{array}{l}\text { eGFR (MDRD), } \mathrm{mL} / \mathrm{min} / 1.73 \\
\mathrm{~m}^{2}\end{array}$ & $53 \pm 29$ & $53 \pm 28$ & $58 \pm 29$ & $62 \pm 30$ & 0.44 \\
\hline Serum sodium, $\mathrm{mEq} / \mathrm{L}^{*}$ & $139(137,141)$ & $140(137,142)$ & $139(136,141)$ & $139(137,141)$ & 0.18 \\
\hline NT-proBNP, pg/mL & $2111(1093,6013)$ & $988(491,2460)$ & $1833(707,4008)$ & $1055(419,2147)$ & $<0.01$ \\
\hline Neprilysin, $\mathrm{ng} / \mathrm{mL}^{*}$ & $1.39(0.74,2.30)$ & $1.51(0.74,2.73)$ & $1.59(0.96,3.14)$ & $1.56(1.82,2.40)$ & 0.16 \\
\hline \multicolumn{6}{|l|}{ Medical treatment } \\
\hline Loop diuretics, n (\%) & $76(88.4)$ & $196(98.0)$ & $202(86.3)$ & $456(91.0)$ & $<0.01$ \\
\hline MRA, n (\%) & $51(59.3)$ & $115(57.5)$ & $120(51.3)$ & $311(62.1)$ & 0.05 \\
\hline Beta-blockers, n (\%) & 78 (90.7) & $170(85.0)$ & $205(87.6)$ & $470(93.8)$ & $<0.01$ \\
\hline ACEI or ARB, n (\%) & $71(82.6)$ & $175(87.5)$ & $204(87.2)$ & $468(93.4)$ & $<0.01$ \\
\hline
\end{tabular}

Table 1. Baseline characteristics across sex and BMI. Continuous variables are expressed as mean \pm 1 standard deviation unless otherwise specified. $A C E I$ angiotensin-converting enzyme inhibitors, $A R B$ angiotensin II receptor blockers, $B M I$ body mass index, COPD chronic obstructive pulmonary disease, $C R T$ cardiac resynchronization therapy, $D M$ diabetes mellitus, $e G F R$ estimated glomerular filtration rate, $H F$ heart failure, ICD implantable cardioverter-defibrillator, $L V E F$ left ventricle ejection fraction, MDRD Modification of Diet in Renal Disease formula, $M R A$ mineralocorticoid receptor antagonist, $N T$-proBNP amino-terminal pro-brain natriuretic peptide, NYHA New York Heart Association, SBP systolic blood pressure, sNEP soluble form of neprilysin. ${ }^{\star}$ Variable expressed as median (interquartile interval).

mortality, the covariates were the same, plus the inclusion of hemoglobin (FP:1) and ST2 (FP:-0.5). Risk estimates are presented as incidence rate ratios (IRRs). We set a two-sided $p<0.05$ as the threshold for significance. All analyses were performed in Stata 15.1 (Stata Statistical Software, Release 15 [2017]; StataCorp LP, College Station, TX, USA). We used the "Bivcnto" Stata module for multivariable bivariate Poisson analyses.

\section{Results}

Baseline characteristics. The mean age was $66 \pm 13$ years, and 286 (28\%) patients were women. Most of the patients had LVEF $<40 \%$ (78.9\%), NYHA class I-II (75.7\%), and ischaemic aetiology (51\%). The median levels of sNEP and NT-proBNP were $0.64 \mathrm{ng} / \mathrm{mL}$ (p25\%-p75\%: 0.39-1.22) and $1248 \mathrm{ng} / \mathrm{L}$ (IQI: 538-2825), respectively. The median BMI was $26.9 \mathrm{~kg} / \mathrm{m}^{2}$ (p25\%-p75\%: 24.4-30.4), and 701 (68.7\%) patients had a BMI $\geq 25 \mathrm{~kg} / \mathrm{m}^{2}$, with a similar distribution in both sexes (women vs. men: $69.9 \%$ vs. $68.2 \%, p=0.585$ ). The number and proportion of patients in the pre-specified categories were as follows: women/BMI $<25 \mathrm{~kg} / \mathrm{m}^{2}, \mathrm{n}=86(8.4 \%)$; women/ $\mathrm{BMI} \geq 25 \mathrm{~kg} / \mathrm{m}^{2}, \mathrm{n}=200(19.6 \%) ; \mathrm{men} / \mathrm{BMI}<25 \mathrm{~kg} / \mathrm{m}^{2}, \mathrm{n}=234(22.9 \%) ;$ and $\mathrm{men} / \mathrm{BMI} \geq 25 \mathrm{~kg} / \mathrm{m}^{2}, \mathrm{n}=501$ (49.1\%). The baseline characteristics across the sex/BMI subgroups are presented in Table 1. Overall, overweight women showed higher prior hypertension, type 2 diabetes, and lower values of NT-proBNP (Table 1).

sNEP and baseline characteristics. Table 2 summarizes the baseline characteristics stratified by sNEP quintiles. Age, history of hypertension, ischaemic etiology, and LVEF $<40 \%$ were inversely associated with the sNEP quintile. In contrast, heart rate was positively associated with the sNEP quintile. We found no significant 
Demographics and medical history

\begin{tabular}{|c|c|c|c|c|c|c|}
\hline Age, years & $69 \pm 11$ & $68 \pm 11$ & $67 \pm 13$ & $64 \pm 14$ & $64 \pm 14$ & $<0.01$ \\
\hline Men, n (\%) & $148(72.2)$ & $154(75.5)$ & $143(70.1)$ & $148(72.5)$ & $142(69.6)$ & 0.69 \\
\hline BMI, $\mathrm{kg} / \mathrm{m}^{2}$ & $27.39 \pm 5.90$ & $27.97 \pm 5.24$ & $27.68 \pm 4.95$ & $27.77 \pm 5.50$ & $27.65 \pm 5.27$ & 0.87 \\
\hline Hypertension, n (\%) & $138(67.3)$ & $132(64.7)$ & $136(66.7)$ & $113(55.4)$ & $118(57.8)$ & 0.04 \\
\hline DM, n (\%) & $70(34.1)$ & $67(32.8)$ & $80(39.2)$ & $68(33.3)$ & $75(36.8)$ & 0.63 \\
\hline Dyslipidemia, n (\%) & $99(48.3)$ & $99(48.5)$ & $93(45.6)$ & $96(47.1)$ & $94(46.1)$ & 0.97 \\
\hline $\begin{array}{l}\text { Current smoker, } \\
\mathrm{n}(\%)\end{array}$ & $28(13.7)$ & $39(19.1)$ & $27(13.2)$ & $43(21.1)$ & $26(12.7)$ & 0.06 \\
\hline $\begin{array}{l}\text { Former smoker, } \\
\mathrm{n}(\%)\end{array}$ & $85(41.5)$ & $88(43.1)$ & $88(43.1)$ & $85(41.7)$ & $80(39.2)$ & 0.93 \\
\hline COPD, n (\%) & $30(14.6)$ & $48(23.5)$ & $32(15.7)$ & $29(14.2)$ & $35(17.2)$ & 0.08 \\
\hline IHD etiology, n (\%) & $124(60.5)$ & $101(49.5)$ & $105(51.5)$ & $96(47.1)$ & 95 (46.6) & 0.03 \\
\hline $\begin{array}{l}\text { HF duration, } \\
\text { months }\end{array}$ & $44.5 \pm 58.0$ & $51.4 \pm 68.7$ & $45.4 \pm 60.9$ & $48.6 \pm 62.8$ & $50.4 \pm 63.2$ & 0.75 \\
\hline ICD, n (\%) & $34(16.6)$ & $19(9.3)$ & $25(12.3)$ & $27(13.2)$ & $31(15.2)$ & 0.23 \\
\hline CRT, n (\%) & $23(11.2)$ & $10(4.9)$ & $20(9.8)$ & $12(5.9)$ & $20(9.8)$ & 0.09 \\
\hline $\begin{array}{l}\text { NYHA class I or II, } \\
\mathrm{n}(\%)\end{array}$ & $163(75.5)$ & $152(74.5)$ & $150(73.5)$ & $158(77.4)$ & $150(73.5)$ & 0.531 \\
\hline LVEF < 40\%, n (\%) & $164(80.0)$ & $170(83.3)$ & $171(83.8)$ & $154(75.5)$ & $147(72.1)$ & 0.013 \\
\hline \multicolumn{7}{|l|}{ Vital signs } \\
\hline Heart rate, bpm & $69 \pm 12$ & $72 \pm 14$ & $73 \pm 14$ & $73 \pm 16$ & $73 \pm 15$ & 0.04 \\
\hline SBP, $\mathrm{mmHg}$ & $127 \pm 25$ & $125 \pm 20$ & $126 \pm 20$ & $128 \pm 24$ & $127 \pm 23$ & 0.73 \\
\hline \multicolumn{7}{|l|}{ Laboratory } \\
\hline Hemoglobin, g/dL & $12.7 \pm 1.7$ & $13.0 \pm 1.9$ & $12.7 \pm 1.9$ & $13.1 \pm 2.0$ & $12.9 \pm 1.8$ & 0.08 \\
\hline $\begin{array}{l}\text { eGFR (MDRD), } \\
\mathrm{mL} / \mathrm{min} / 1.73 \mathrm{~m}^{2}\end{array}$ & $59 \pm 27$ & $60 \pm 31$ & $56 \pm 29$ & $59 \pm 34$ & $59 \pm 26$ & 0.79 \\
\hline $\begin{array}{l}\mathrm{eGFR}<60 \mathrm{~mL} / \\
\mathrm{min} / 1.73 \mathrm{~m}^{2}, \mathrm{n}(\%)\end{array}$ & $117(57.1)$ & $108(52.9)$ & $125(61.3)$ & $111(54.4)$ & $113(55.4)$ & 0.49 \\
\hline $\begin{array}{l}\text { Serum sodium, } \\
\mathrm{mEq} / \mathrm{L}\end{array}$ & $139 \pm 3$ & $139 \pm 4$ & $139 \pm 3$ & $139 \pm 3$ & $139 \pm 3$ & 0.16 \\
\hline hsTNT, ng/L* & $28(14,40)$ & $22(13,37)$ & $25(11,45)$ & $26(13,43)$ & $25(12,38)$ & 0.64 \\
\hline $\begin{array}{l}\text { NT-proBNP, pg/ } \\
\text { mL* }^{*}\end{array}$ & $1310(594,2858)$ & $1034(540,2635)$ & $1318(489,2967)$ & $1484(550,3114)$ & $1165(535,2725)$ & 0.73 \\
\hline Neprilysin, ng/mL $L^{*}$ & $0.25(0.25,0.26)$ & $0.46(0.39,0.51)$ & $0.64(0.60,0.70)$ & $0.98(0.84,1.22)$ & $3.25(2.00,8.92)$ & $<0.01$ \\
\hline \multicolumn{7}{|l|}{ Medical treatment } \\
\hline $\begin{array}{l}\text { Loop diuretics, } \\
\text { n (\%) }\end{array}$ & $184(89.8)$ & $191(93.6)$ & $188(92.2)$ & $181(88.7)$ & $186(91.2)$ & 0.44 \\
\hline MRA, n (\%) & $119(58.0)$ & $107(52.5)$ & $122(59.8)$ & $123(60.3)$ & $126(61.8)$ & 0.36 \\
\hline ACEI or ARB, n (\%) & $61(29.8)$ & $43(21.1)$ & $59(28.9)$ & $55(27.0)$ & $53(26.0)$ & 0.30 \\
\hline Beta-blockers, n (\%) & $187(91.2)$ & $189(92.6)$ & $183(89.7)$ & $180(88.2)$ & $179(87.7)$ & 0.44 \\
\hline
\end{tabular}

Table 2. Baseline characteristics across sNEP quintiles. Continuous variables are expressed as mean \pm 1 standard deviation unless otherwise specified. $A C E I$ angiotensin-converting enzyme inhibitors, $A R B$ angiotensin II receptor blockers, $B M I$ body mass index, COPD chronic obstructive pulmonary disease, $C R T$ cardiac resynchronization therapy, $D M$ diabetes mellitus, $e G F R$ estimated glomerular filtration rate, $H F$ heart failure, ICD implantable cardioverter-defibrillator, $L V E F$ left ventricle ejection fraction, $M D R D$ Modification of Diet in Renal Disease formula, $M R A$ mineralocorticoid receptor antagonist, NT-proBNP amino-terminal probrain natriuretic peptide, NYHA New York Heart Association, SBP systolic blood pressure, $s N E P$ soluble form of neprilysin. ${ }^{\star}$ Variable expressed as median (interquartile interval).

differences in sex, BMI, HF duration, NYHA functional class, systolic blood pressure, hemoglobin, sodium, renal function markers, or NT-proBNP among sNEP quartiles.

The median sNEP did not differ between women and men $[0.68 \mathrm{ng} / \mathrm{mL}$ (p25\%-p75\%: 0.41-1.35), vs. $0.63 \mathrm{ng} /$ $\mathrm{mL}$ (IQI: $0.39-1.19), p=0.377$ ], and BMI $\geq 25 \mathrm{~kg} / \mathrm{m}^{2}$ versus $<25 \mathrm{~kg} / \mathrm{m}^{2}$ [0.64 ng/mL (IQI: $0.41-1.23$ ) vs. $0.65 \mathrm{ng} /$ $\mathrm{mL}$ (IQI: $0.36-1.1), p=0.560)$. Similarly, the sNEP distribution did not differ across sex and BMI categories [women/BMI $<25 \mathrm{~kg} / \mathrm{m}^{2}: 0.72 \mathrm{ng} / \mathrm{mL}$ (IQI: 0.44-134); women/BMI $\geq 25 \mathrm{~kg} / \mathrm{m}^{2}: 0.66 \mathrm{ng} / \mathrm{mL}$ (IQI: 0.37-1.35); men $/ \mathrm{BMI}<25 \mathrm{~kg} / \mathrm{m}^{2}: 0.63 \mathrm{ng} / \mathrm{mL}$ (IQI: $0.32-1.04$ ); men $/ \mathrm{BMI} \geq 25 \mathrm{~kg} / \mathrm{m}^{2}: 0.64 \mathrm{ng} / \mathrm{mL}$ (IQI: 0.42-1.22); $p=0.297$ ].

Follow-up. Over a median follow-up of 6.65 years [p25\%-p75\%:2.83-10.25], 702 (68.76\%) patients died. A total of 959 HF-hospitalizations were recorded in 406 patients (40\%). Of these, 231 patients (56.9\%) had two or more admissions. 

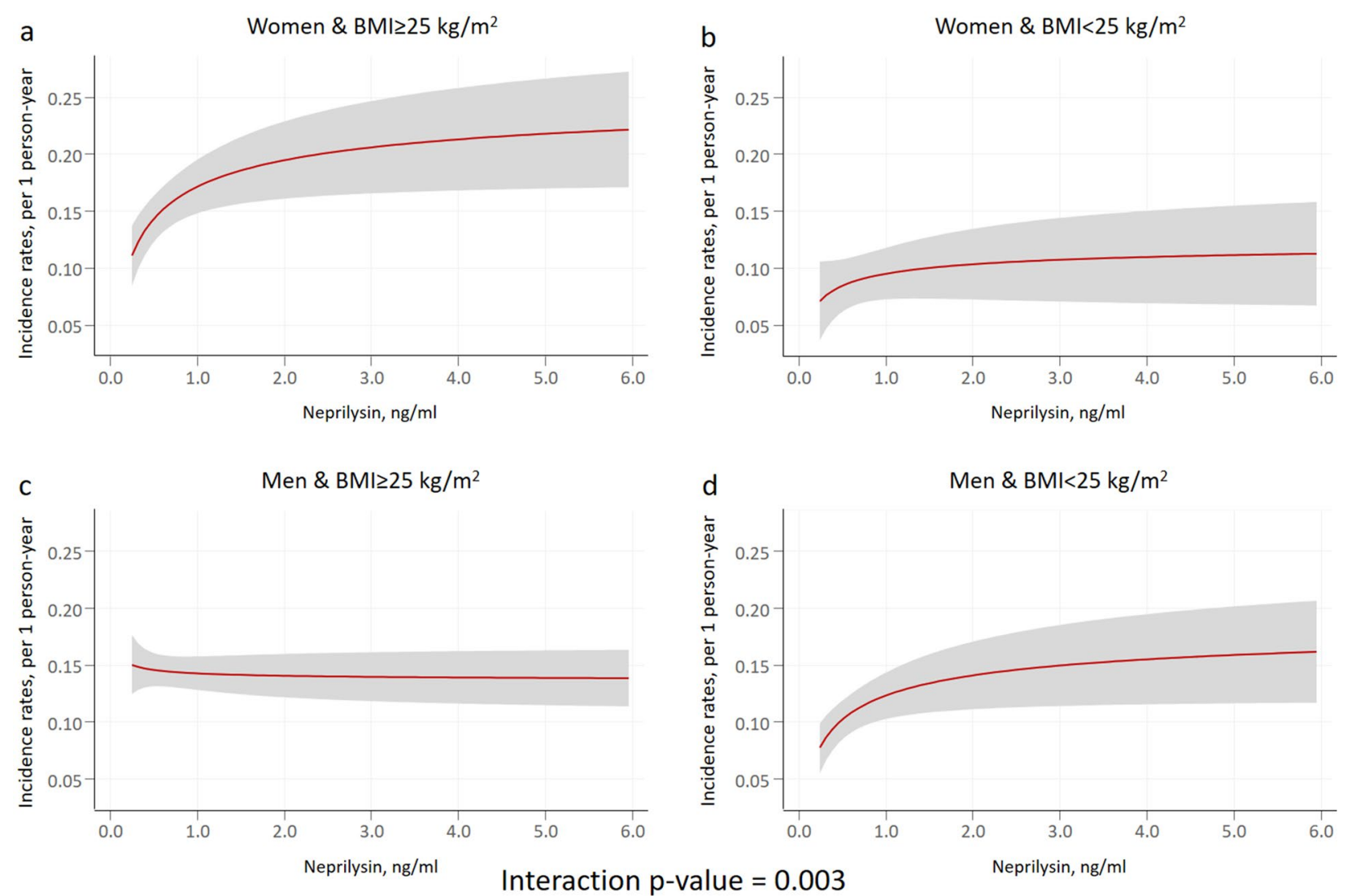

Figure 1. Soluble neprilysin and heart failure admission rates among sex and body mass index categories. Estimates were adjusted for age, HF duration, BMI, NYHA III versus I-II, ischaemic heart disease, systolic blood pressure, heart rate, eGFR, sodium, high-sensitivity troponin T, LVEF, and treatment with ACEI/ARB, beta-blockers, MRA, or loop diuretics. ACEI: angiotensin-converting enzyme inhibitors; ARB: angiotensin II receptor blockers; BMI: body mass index; eGFR: estimated glomerular filtration rate; HF: heart failure; LVEF: left ventricle ejection fraction; MRA: mineralocorticoid receptor antagonist; NT-proBNP: amino-terminal proB-type natriuretic peptide; NYHA: New York Heart Association.

HF hospitalization risk and sex/BMI categories. The risk for HF hospitalization significantly differed across sex-BMI $\geq 25 \mathrm{~kg} / \mathrm{m}^{2}$ categories (overall $p$-value $<0.001$ ). However, the two comparisons of interest (women vs. men across BMI groups) were not significant. The IRRs for women versus men when BMI $<25 \mathrm{~kg} /$ $\mathrm{m}^{2}$ and $\geq 25 \mathrm{~kg} / \mathrm{m}^{2}$ were $0.78,95 \% \mathrm{CI}=0.59-1.04 ; p=0.092$, and $1.08,95 \% \mathrm{CI}=0.92-1.28 ; p=0.354$, respectively.

sNEP and risk of HF hospitalization. sNEP was a significant predictor of HF hospitalizations, either when modeled (as the main effect) continuously [FP-0.5] $(p=0.001)$ or in quintiles (omnibus $p=0.016$ ). Compared to patients in the lower sNEP quintile, patients in the upper quintile had an increased risk of HF hospitalizations (IRR $=1.29 ; 95 \% \mathrm{CI}: 1.06-1.58 ; p=0.013$ ). The incidence rates of this endpoint along the continuum of sNEP across the evaluated subgroups are shown in Fig. 1 . The interaction analysis revealed a differential prognostic effect of sNEP (modeled as continuous with FP [-0.5]) across sex and BMI categories for HF hospitalizations ( $p$-value for interaction $=0.003$ ). Indeed, the sNEP trajectory showed a positive and non-linear association in women with BMI $\geq 25 \mathrm{~kg} / \mathrm{m}^{2}$ compared to the men counterpart ( $\left.p=0.039\right)$ (Figs. 1 and $2 \mathrm{a}$ ). Differences in gender were not present when $\mathrm{BMI}<25 \mathrm{~kg} / \mathrm{m}^{2}(p=0.078)$ (Figs. 1 and $2 \mathrm{~b}$ ). Table 3 shows the estimates of risk (women vs. men) for different values of sNEP across BMI status.

A subgroup analysis stratifying the samples across LVEF $(\leq 40 \%$ and $>40 \%)$, with the same multivariate setting, suggests that the direction and magnitude of the excess risk attributable to higher sNEP in women with $\mathrm{BMI} \geq 25 \mathrm{~kg} / \mathrm{m}^{2}$ were found in both subgroups (Supplementary Fig. 1).

In a sensitivity analysis, we confirmed the differential sNEP gradient of risk across sex and BMI strata when BMI was categorized by the median value (Supplementary Fig. 2).

sNEP and mortality risk-interaction analysis. In multivariable analysis, sNEP (main effect) (modeled as continuous with FP [-0.5]) was not associated with mortality risk $(p=0.241)$. The prognostic effect of sNEP on mortality did not significantly differ across sex and BMI categories ( $p$-value for interaction $=0.072$; Fig. 3). Indeed, any of the two comparisons of interest (women vs. men in the two BMI groups (BMI $\geq 25 \mathrm{~kg} /$ $\mathrm{m}^{2}$ vs. BMI $\left.<25 \mathrm{~kg} / \mathrm{m}^{2}\right)$ were not significant $(p=0.563$ and $p=0.064$, respectively) (Figs. $4 \mathrm{a}$, b). Worth noting, 

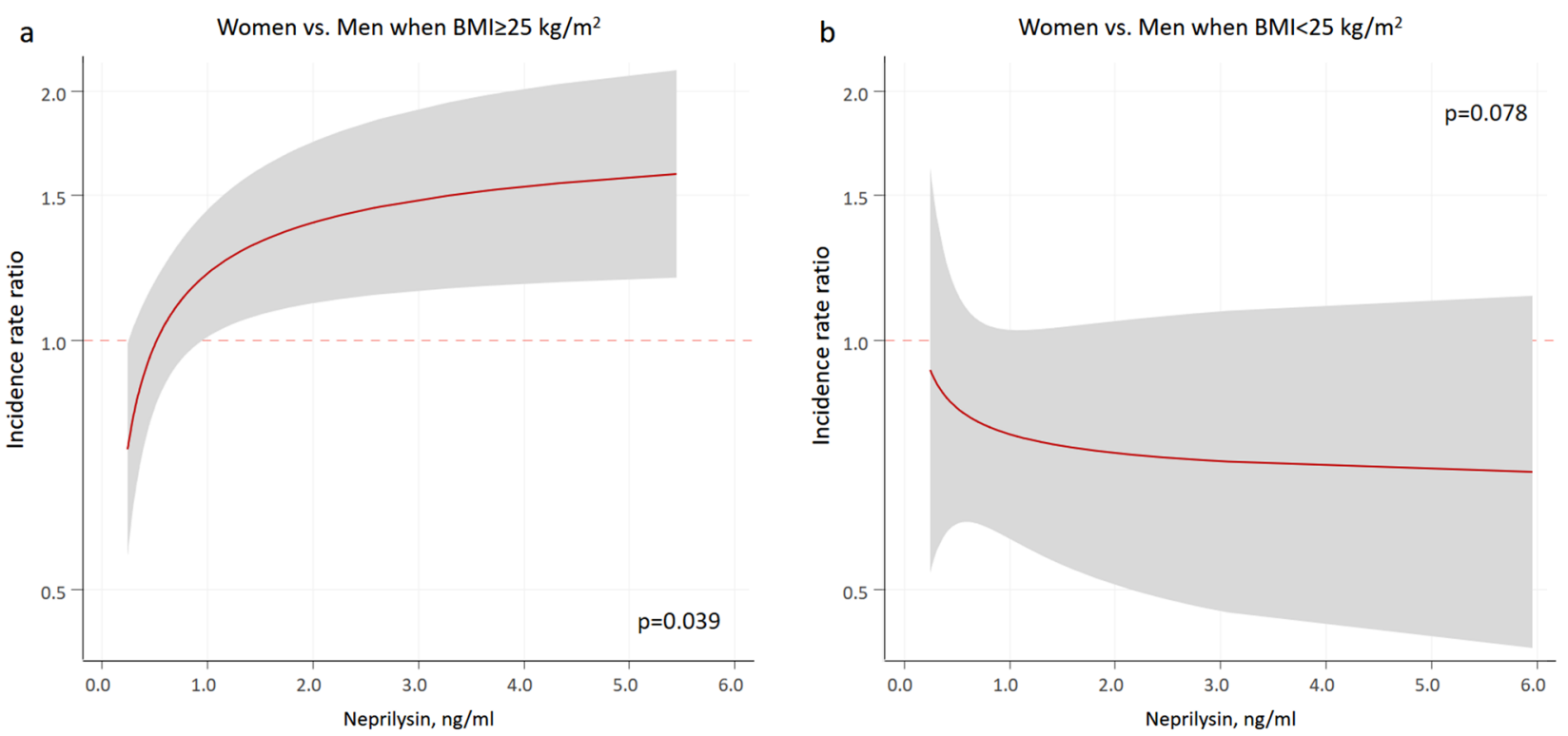

Figure 2. Soluble neprilysin and excess hospitalization risk in women versus men across body mass index values. BMI: body mass index.

\begin{tabular}{|c|c|c|c|c|}
\hline & \multicolumn{2}{|c|}{ BMI $\geq 25 \mathrm{~kg} / \mathrm{m}^{2}$} & \multicolumn{2}{|c|}{$\mathrm{BMI}<25 \mathrm{~kg} / \mathrm{m}^{2}$} \\
\hline & \multicolumn{2}{|c|}{ Women vs. men } & \multicolumn{2}{|c|}{ Women vs. men } \\
\hline & IRR & $95 \% \mathrm{CI}$ & IRR & 95\% CI \\
\hline \multicolumn{5}{|c|}{ HF-hospitalizations } \\
\hline $0.25 \mathrm{ng} / \mathrm{mL}^{\mathrm{a}}$ & 0.78 & $0.60-1.02$ & 0.91 & $0.53-1.56$ \\
\hline $0.39 \mathrm{ng} / \mathrm{mL}^{\mathrm{b}}$ & 0.91 & $0.74-1.11$ & 0.85 & $0.59-1.24$ \\
\hline $0.64 \mathrm{ng} / \mathrm{mL}^{\mathrm{c}}$ & 1.06 & $0.90-1.26$ & 0.80 & $0.60-1.07$ \\
\hline $1.22 \mathrm{ng} / \mathrm{mL}^{\mathrm{d}}$ & 1.26 & $1.05-1.53$ & 0.76 & \begin{tabular}{|l|}
$0.56-1.03$ \\
\end{tabular} \\
\hline $3.24 \mathrm{ng} / \mathrm{mL}^{\mathrm{e}}$ & 1.50 & $1.16-1.94$ & 0.71 & \begin{tabular}{|l|}
$0.47-1.10$ \\
\end{tabular} \\
\hline \multicolumn{5}{|c|}{ All-cause mortality } \\
\hline $0.25 \mathrm{ng} / \mathrm{mL}^{\mathrm{a}}$ & 0.62 & $0.44-0.84$ & 0.90 & $0.63-1.41$ \\
\hline $0.39 \mathrm{ng} / \mathrm{mL}^{\mathrm{b}}$ & 0.71 & $0.57-0.90$ & 0.84 & \begin{tabular}{|l|}
$0.59-1.13$ \\
\end{tabular} \\
\hline $0.64 \mathrm{ng} / \mathrm{mL}^{\mathrm{c}}$ & 0.83 & $0.68-1.02$ & 0.80 & $0.62-1.03$ \\
\hline $1.22 \mathrm{ng} / \mathrm{mL}^{\mathrm{d}}$ & 0.97 & $0.76-1.22$ & 0.78 & \begin{tabular}{|l|}
$0.54-1.06$ \\
\end{tabular} \\
\hline $3.24 \mathrm{ng} / \mathrm{mL}^{\mathrm{e}}$ & 1.12 & $0.81-1.52$ & 0.72 & $0.40-1.11$ \\
\hline
\end{tabular}

Table 3. sNEP and adjusted risk of adverse outcomes across sex and BMI. BMI body mass index, $s N E P$ serum

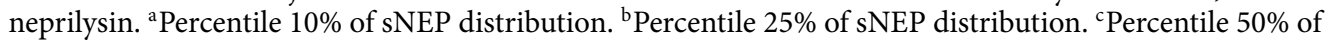
sNEP distribution. ${ }^{\mathrm{d}}$ Percentile $75 \%$ of sNEP distribution. ${ }^{~}$ Percentile $90 \%$ of sNEP distribution.

however, is that the sNEP depicted trajectories are almost identical to those found for HF hospitalization. The estimates of risk (women vs. men) for different values of sNEP are presented in Table 3.

\section{Discussion}

In this large cohort of ambulatory patients with chronic HF and predominantly reduced LVEF, sNEP predicted the risk of HF hospitalization, especially in overweight women. Our data suggested that sNEP activity may be influenced by sex and excess adipose tissue.

The following explanations have been postulated to backed-up such interaction. Mature adipocytes are known to produce and express neprilysin ${ }^{16}$, and obese individuals have increased levels of sNEP in proportion to their body mass ${ }^{17}$. In the setting of HF, a leptin-aldosterone-neprilysin axis has been proposed ${ }^{7}$. Packer postulated that obesity aggravates the deleterious interaction of leptin with the renin-angiotensin-aldosterone system and the renal sympathetic system leading to overactivity of neprilysin and, thus, deficiency in endogenous natriuretic peptides in these patients ${ }^{7}$. Also, it has been postulated that obesity-driven aldosterone and neprilysin overactivity generate a vicious circle that further stimulates adipogenesis, adipocyte dysfunction, and adipose tissue inflammation, thereby enhancing this deleterious feedback loop $p^{7,18}$. 

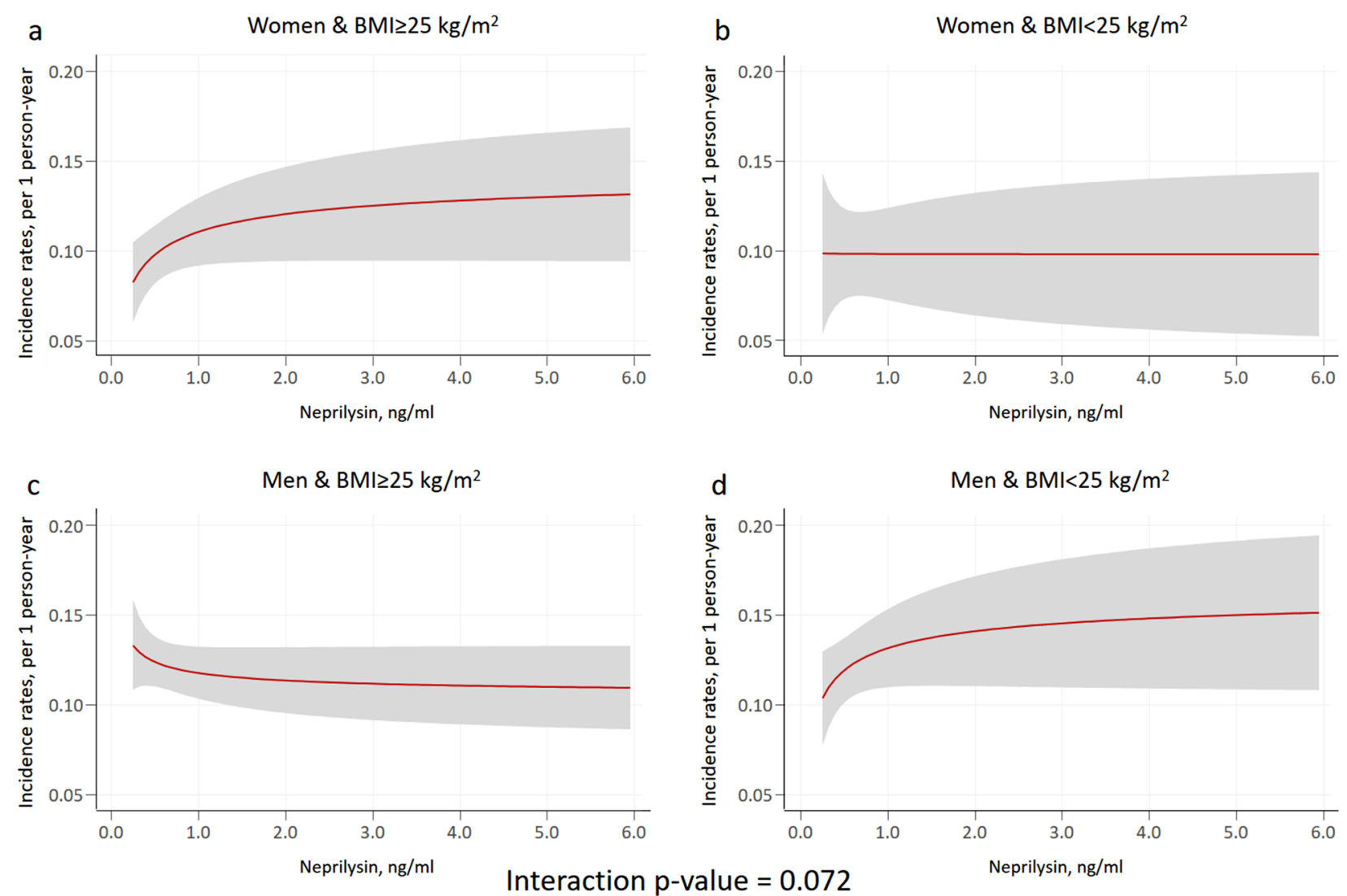

Figure 3. Soluble neprilysin and mortality rates among sex and body mass index categories. Estimates of risk were adjusted for age, HF duration, BMI, NYHA III versus I-II, ischaemic heart disease, systolic blood pressure, heart rate, eGFR, sodium, high-sensitivity troponin T, haemoglobin, ST2, LVEF, and treatment with ACEI/ ARB, beta-blockers, MRA, or loop diuretics. ACEI: angiotensin-converting enzyme inhibitors; ARB: angiotensin II receptor blockers; BMI: body mass index; eGFR: estimated glomerular filtration rate; HF: heart failure; LVEF: left ventricle ejection fraction; MRA: mineralocorticoid receptor antagonist; NYHA: New York Heart Association.
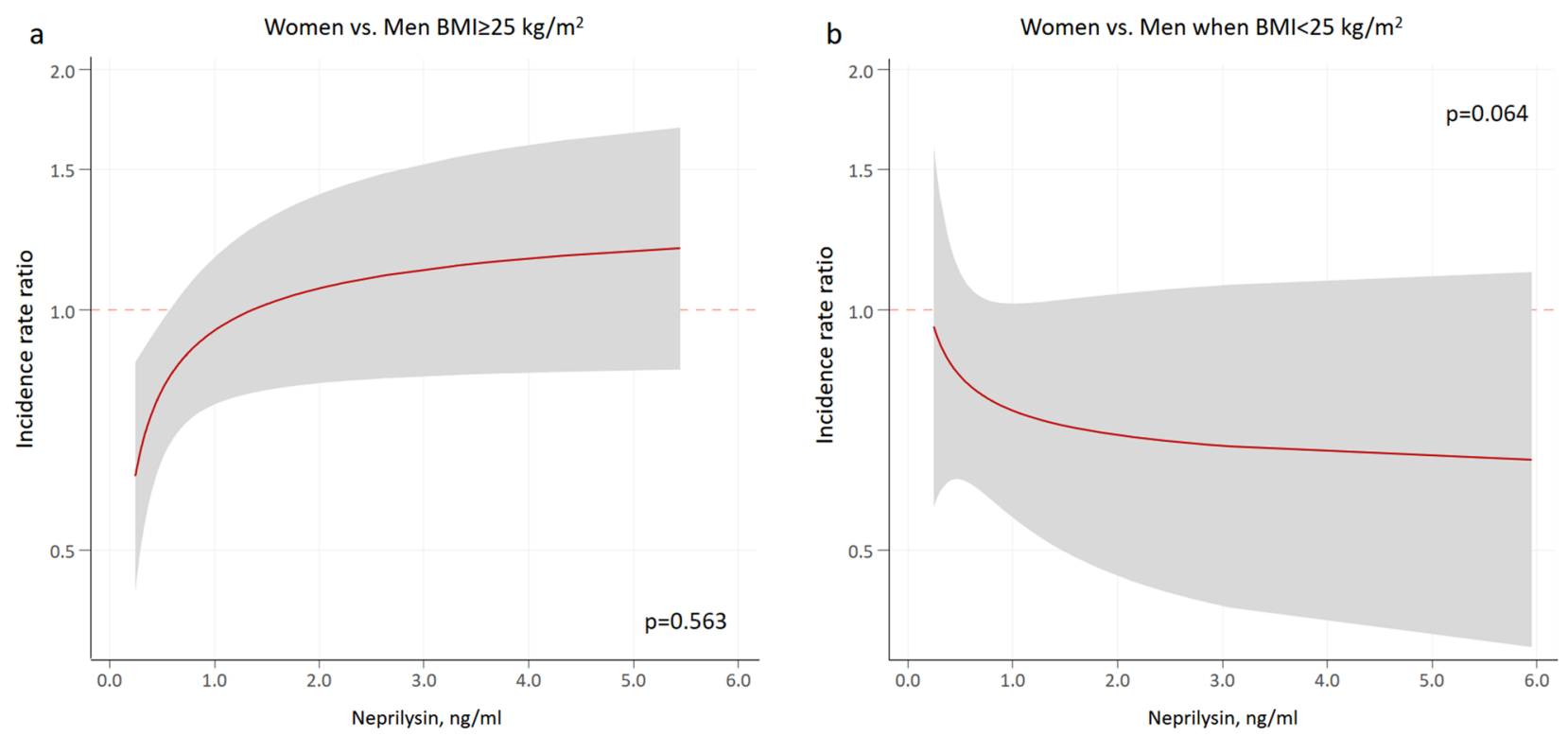

Figure 4. Soluble neprilysin and excess mortality risk in women versus men across body mass index values. BMI: body mass index. 
There is no clear explanation about the positive association between sNEP and the risk of HF hospitalizations in overweight women, particularly in the absence of significantly elevated levels of sNEP in this subgroup. Complicating this issue is also the controversy about the utility of sNEP as a proxy of NEP activity ${ }^{7}$. We speculate that sNEP may better reflect NEP activity in the subset of overweight women, a hypothesis that is endorsed by some findings regarding lower medians of NT-proBNP in this group. On this same line, we recently proposed the concept of "natriuretic peptide availability" based on the balance between natriuretic peptide synthesis and degradation (which is mediated by neprilysin) ${ }^{19}$. Elaborating upon this concept, the neprilysin/NT-proBNP ratio (or alternative formulas integrating both biomarkers) could serve as an alternative surrogate for neprilysin activity, which would be particularly useful in obese patients especially given the complexity of measuring neprilysin activity itself.

The clinical association between sNEP and sex reported here, and in the PARAGON-HF trial of patients with $\mathrm{HF}$ and preserved ejection fraction ${ }^{4}$, raises the question as to whether these findings reflect association or causation $^{20}$. Following the framework of the leptin-aldosterone-neprilysin axis, Packer postulated that women and the elderly have high levels of aldosterone, leptin, and neprilysin, with lower endogenous natriuretic peptide levels ${ }^{7}$. A biological difference cannot be ruled out, as oestrogens have been reported to up-regulate the expression of neprilysin ${ }^{21}$.

Nevertheless, most patients enrolled in HF studies, including ours, are post-menopausal women; as such, oestrogen deficiency would yield low sNEP levels. We humbly acknowledge the possibility of dark corners in the complex regulation and counter-regulation of the neuro-hormonal axis in HF that escape our understanding. Importantly, the findings observed in the setting of HF do not concur with those of the general population, not affected by neurohormonal activation. A recent report of 1536 participants from Olmsted County, Minnesota, did not find significant associations between sNEP levels and age, sex, or renal function ${ }^{12}$.

Study limitations. The present study has some limitations. First, our study included a Mediterranean cohort of predominantly white men and reduced systolic function HF patients, and thereby limiting the extrapolation of our results to other ethnicities or patients with $\mathrm{HF}$ and preserved ejection fraction. Also, the proportion of obese patients in this cohort precluded exploring whether this sex-BMI differential effect of sNEP may be even greater in obese patients. Second, analytical issues regarding sNEP measurement must be resolved before its routine implementation in daily clinical practice. Third, the lack of serial assessment of sNEP and BMI status during the follow-up precluded inferring how the association here found may describe a dynamic pattern. Fourth, the low proportion of patients/events in some comparisons, especially in patients with LVEF $\geq 40 \%$, decreased the statistical power and increasing the possibility of a type II error. Finally, with the present data, we cannot explore the potential role of sNEP for tailoring sacubitril/valsartan.

\section{Conclusions}

In chronic HF with predominant left systolic dysfunction, high sNEP levels in overweight women predicted an increased risk for recurrent HF hospitalizations. Further studies are warranted to explore whether sNEP levels can be used to tailor angiotensin receptor blocker and neprilysin inhibitor therapy. The more knowledge we gain in HF's pathobiology and its management, the greater the need for a precision medicine approach centered on disease biology with biomarkers as surrogates.

Received: 7 November 2020; Accepted: 2 March 2021

Published online: 15 March 2021

\section{References}

1. Bayes-Genis, A., Barallat, J. \& Richards, A. M. A test in context: neprilysin: function, inhibition, and biomarker. J. Am. Coll. Cardiol. 68, 639-653 (2016).

2. McMurray, J. J. et al. Angiotensin-neprilysin inhibition versus enalapril in heart failure. N. Engl. J. Med. 371, 993-1004 (2014).

3. Ponikowski, P. et al. 2016 ESC guidelines for the diagnosis and treatment of acute and chronic heart failure: the task force for the diagnosis and treatment of acute and chronic heart failure of the European Society of Cardiology (ESC) developed with the special contribution of the Heart Failure Association (HFA) of the ESC. Eur. Heart J. 37, 2129-2200 (2016).

4. Solomon, S. D. et al. Angiotensin-neprilysin inhibition in heart failure with preserved ejection fraction. N. Engl. J. Med. 381, 1609-1620 (2019).

5. McMurray, J. J. V. et al. Effects of sacubitril-valsartan versus valsartan in women compared with men with heart failure and preserved ejection fraction: insights from PARAGON-HF. Circulation 141, 338-351 (2020).

6. Solomon, S. D. et al. Sacubitril/valsartan across the spectrum of ejection fraction in heart failure. Circulation 141, 352-361 (2020).

7. Packer, M. Leptin-aldosterone-neprilysin axis: identification of its distinctive role in the pathogenesis of the three phenotypes of heart failure in people with obesity. Circulation 137, 1614-1631 (2018).

8. Bayes-Genis, A. et al. Soluble neprilysin is predictive of cardiovascular death and heart failure hospitalization in heart failure patients. J. Am. Coll. Cardiol. 65, 657-665 (2015).

9. Bayés-Genís, A. et al. Prognostic value and kinetics of soluble neprilysin in acute heart failure: a pilot study. JACC Heart Fail. 3 , 641-644 (2015).

10. Núñez, J. et al. Serum neprilysin and recurrent admissions in patients with heart failure. J. Am. Heart Assoc. 6, e005712 (2017).

11. Núñez, J. et al. Serum neprilysin and recurrent hospitalizations after acute heart failure. Int. J. Cardiol. 220, 742-744 (2016).

12. Reddy, Y. N. V. et al. Soluble neprilysin in the general population: clinical determinants and its relationship to cardiovascular disease. J. Am. Heart Assoc. 8, e012943 (2019).

13. Goliasch, G. et al. Soluble neprilysin does not correlate with outcome in heart failure with preserved ejection fraction. Eur. J. Heart Fail. 18, 89-93 (2016).

14. Xu, X. \& Hardin, J. W. Regression models for bivariate count outcomes. Stata J. 16, 301-315 (2016).

15. Keys, A. et al. Indices of relative weight and obesity. J. Chronic Dis. 25, 329-343 (1972). 
16. Schling, P. \& Schäfer, T. Human adipose tissue cells keep tight control on the angiotensin II levels in their vicinity. J. Biol. Chem. 277, 48066-48075 (2002).

17. Standeven, K. F. et al. Neprilysin, obesity and the metabolic syndrome. Int. J. Obes. (Lond.) 35, 1031-1040 (2011).

18. Achike, F. I. et al. Obesity, metabolic syndrome, adipocytes and vascular function: a holistic viewpoint. Clin. Exp. Pharmacol. Physiol. 38, 1-10 (2011).

19. Gommans, D. H., Bayés-Genís, A. \& van Kimmenade, R. R. Putting together the pieces of the natriuretic peptide puzzle. JACC Heart Fail. 4, 670-673 (2016).

20. Bozkurt, B. \& Ezekowitz, J. Substance and substrate: LVEF and sex subgroup analyses of PARAGON-HF and PARADIGM-HF trials. Circulation 141, 362-366 (2020).

21. Liang, K. et al. Estrogen stimulates degradation of beta-amyloid peptide by up-regulating neprilysin. J. Biol. Chem. 285, 935-942 (2010).

\section{Author contributions}

J.N. and A.B.-G.: conceptualization, data curation, funding acquisition, methodology, formal analysis, project administration, supervision, validation, visualization, and writing. E.N.: data curation, methodology, and formal analysis. E.R.-L.: data curation, validation, and visualization. G.M.: methodology, data curation, validation, visualization, and writing. J.B.: data curation, validation, and visualization. V.B. and J.S.: funding acquisition, methodology, and project administration. A.A. and M.E.: validation, visualization, and writing. J.L.: data curation, funding acquisition, and methodology. All authors reviewed and approved the final version of the manuscript. J.N. and A.B-G. are responsible for the overall content as guarantors.

\section{Funding}

The study was funded by Grants from CIBER Cardiovascular (16/11/00420, 16/11/00403), and Instituto de Salud Carlos III (PIE15/00013).

\section{Competing interests}

Julio Núñez has received speaker fees from Novartis, Vifor Pharma, Boehringer Ingelheim, Astra Zeneca, Rovi, and Novonordisk. The other authors have no disclosures.

\section{Additional information}

Supplementary Information The online version contains supplementary material available at https://doi. org/10.1038/s41598-021-85490-1.

Correspondence and requests for materials should be addressed to J.N.

Reprints and permissions information is available at www.nature.com/reprints.

Publisher's note Springer Nature remains neutral with regard to jurisdictional claims in published maps and institutional affiliations.

(c) (1) Open Access This article is licensed under a Creative Commons Attribution 4.0 International License, which permits use, sharing, adaptation, distribution and reproduction in any medium or format, as long as you give appropriate credit to the original author(s) and the source, provide a link to the Creative Commons licence, and indicate if changes were made. The images or other third party material in this article are included in the article's Creative Commons licence, unless indicated otherwise in a credit line to the material. If material is not included in the article's Creative Commons licence and your intended use is not permitted by statutory regulation or exceeds the permitted use, you will need to obtain permission directly from the copyright holder. To view a copy of this licence, visit http://creativecommons.org/licenses/by/4.0/.

(C) The Author(s) 2021 